\title{
Picolinyl Ester Fragmentation Mechanism Studies with Application to the Identification of Acylcarnitine Acyl Groups Following Transesterification
}

\author{
Shuming Yang, Paul Minkler, ${ }^{*}$ and Charles Hoppel* \\ Department of Medicine and Pharmacology, Case School of Medicine, Case Western Reserve University, \\ Cleveland, Ohio, USA
}

\author{
Kou-Yi Tserng* \\ Department of Nutrition, Case School of Medicine, Case Western Reserve University, Cleveland, Ohio, USA
}

\begin{abstract}
Acyl picolinyl esters provide excellent data to identify the structures of acyl groups. However, the mechanisms for the formation of fragment ions from picolinyl esters are unsettled. Proposed structures for fragment ions have focused on long-chain groups and may not accommodate results from medium- and short-chain acyl groups. Using deuterium-labeled organic acids, we have investigated the mechanisms for the formation of fragment ions. Based on these studies, we propose a new mechanism that is consistent with the experimental data. We then tested the mechanisms by analyzing selected acylcarnitines. Transesterification of acylcarnitines was performed by reaction with 3-pyridylcarbinol and potassium tert-butoxide in dichloromethane to produce acyl picolinyl esters. The picolinyl esters were separated and detected by gas chromatography/electron ionization-mass spectrometry. Each mass spectrum contained a series of peaks with $\mathrm{m} / \mathrm{z}$ differences of 12,13 , or $14 \mathrm{u}$ depending on the acyl group's chemical structure. The position of an unsaturated bond or branched methyl in the acyl group of acylcarnitine can be readily determined. (J Am Soc Mass Spectrom 2006, 17, 1620-1628) (C) 2006 American Society for Mass Spectrometry
\end{abstract}

$\mathrm{F}$ atty acid picolinyl esters have been used to characterize the chemical structure of fatty acids because the position of double bonds or branched methyl groups can be deduced from the fragment ion patterns produced by electron ionization-mass spectrometry (EI-MS) [1, 2]. Mechanisms have been proposed by Harvey [3] and by Hamilton and Christie [4] in which the fragment ions of an acyl picolinyl ester result from the interaction of alkyl hydrogens with the ionized nitrogen on the pyridine ring. The mechanism invoking a McLafferty rearrangement, proposed by Harvey for the formation of the fragment ions from long-chain acyl groups, is not supported by data from Christie's laboratory [4, 5]. Furthermore, these mechanistic studies have not been conducted using either short- or medium-chain acyl groups.

Acyl group identification is an important component in many analytical methods. Several reports detail procedures to identify the acyl groups of acylcarnitines.

Published online August 9, 2006

Address reprint requests to Dr. C. Hoppel, Department of Medicine and Pharmacology, Case School of Medicine, Case Western Reserve University, Cleveland, OH 44106, USA. E-mail: charles.hoppel@case.edu

* Also at the Medical Research Service, Louis Stokes Department of Veterans Affairs Medical Center, Cleveland, $\mathrm{OH} 44106$, USA.
Lowes and Rose [6, 7] reported that by converting acylcarnitines into acyloxylactones, partial, but not complete, structural information of the acyl group was obtained. Libert et al. [8-10] used a procedure whereby acylcarnitines were extracted from urine and hydrolyzed, followed by conversion of the organic acids into acyl picolinyl esters. These acyl picolinyl esters were then characterized by GC/MS, which provided better structural data than in the case of acyloxylactones. However, the presence of organic acids in urine makes questionable the interpretation of the source of acyl groups as being exclusively from acylcarnitines. Although acyl picolinyl esters usually are produced from organic acids, transesterification has been reported to produce acyl picolinyl esters from lipids by direct reaction with 3-pyridylcarbinol, thus eliminating the need to hydrolyze the lipids first [11].

In this paper, we propose mechanisms for the formation of the fragment ions of acyl picolinyl esters based on experiments using deuterium-labeled organic acids. We applied these results to the characterization of acyl groups of several acylcarnitine species following their transesterification to acyl picolinyl esters. The transesterification was performed by reacting acylcarnitines with 3-pyridylcarbinol and potassium tert- 
butoxide in dichloromethane to form picolinyl esters in one step. The resulting picolinyl ester products were analyzed using GC/EI/MS. The position of a doublebond or a branched methyl group in an acylcarnitine can be readily deduced from the EI/MS spectrum of the corresponding picolinyl ester.

\section{Experimental}

\section{Reagents}

Potassium tert-butoxide (1.0 M solution in tetrahydrofuran), anhydrous dichloromethane, and 3-pyridylcarbinol were purchased from Aldrich (St. Louis, MO). Acetylcarnitine, propionylcarnitine, butyrylcarnitine, isobutyrylcarnitine, pentanoylcarnitine, hexanoylcarnitine, heptanoylcarnitine, octanoylcarnitine, 4-methyloctanoylcarnitine, nonanoylcarnitine, decanoylcarnitine, lauroylcarnitine, myristoylcarnitine, palmitoylcarnitine, 2-methyl-hexadecanoylcarnitine, and stearoylcarnitine were synthesized as described [12] from their corresponding acylchlorides, obtained from Aldrich. Trans2-acylcarntines (C8, C10, C12, C14, and C16), trans-4octenoylcarnitine, and cis-4-octenoylcarnitine were synthesized in our laboratory (manuscript in preparation). Pentanoic-2,2- $\mathrm{d}_{2}$ acid, pentanoic-3,3- $\mathrm{d}_{2}$ acid, pentanoic- $4,4-\mathrm{d}_{2}$ acid, pentanoic-5,5,5- $\mathrm{d}_{3}$ acid, and octanoic$8,8,8-\mathrm{d}_{3}$ acid were purchased from $\mathrm{C} / \mathrm{D} / \mathrm{N}$ Isotopes (Quebec, Canada). Propionic-2,2- $\mathrm{d}_{2}$ acid and propionic3,3,3- $\mathrm{d}_{3}$ acid were purchased from Cambridge Isotope Laboratories (Andover, MA). Other chemicals used were of reagent grade.

\section{Gas Chromatography}

GC/EI/MS analyses were performed on a system consisting of an HP6890 GC, an HP5973 mass spectrometer, and an HP7683 autosampler. Samples $(1 \mu \mathrm{L})$ were injected into a split/splitless injector set at $280^{\circ} \mathrm{C}$ under the splitless mode. The picolinyl esters were resolved on a capillary column of $30 \mathrm{~m} \times 0.25 \mathrm{~mm} \times 0.25 \mu \mathrm{m}$ (HP-5ms). The oven temperature was programmed from 80 to $150{ }^{\circ} \mathrm{C}$ at a rate of $30^{\circ} \mathrm{C} \mathrm{min}{ }^{-1}$, then from 150 to $280^{\circ} \mathrm{C}$ at the rate of $5^{\circ} \mathrm{C} / \mathrm{min}$, and held at $280^{\circ} \mathrm{C}$ for $10 \mathrm{~min}$. Helium was used as the carrier gas at the flow rate of $1.4 \mathrm{~mL} / \mathrm{min}$. The mass spectrometer was operated in the electron ionization mode at $70 \mathrm{eV}$ with a source temperature of $230^{\circ} \mathrm{C}$, and spectra were acquired using a scan function from $\mathrm{m} / \mathrm{z} 50$ to 450 .

Analysis by GC/FID was performed on a system consisting of an HP5890 GC and an HP7673 autosampler. Samples $(1 \mu \mathrm{L})$ were injected into a split/splitless injector set at $280^{\circ} \mathrm{C}$ under splitless mode. The FID detector was set at $280{ }^{\circ} \mathrm{C}$ and hydrogen and compressed air were used for signal generation. A capillary column of $30 \mathrm{~m} \times 0.25 \mathrm{~mm} \times 0.25 \mu \mathrm{m}$ (ZB-5) was used. The same oven temperature program as in GC/MS was employed. Helium was used as the carrier gas at a flow rate of $1.0 \mathrm{~mL} / \mathrm{min}$. The peaks in chromatogram were integrated using the HP Chemstation software.

\section{Synthesis of Deuterium-Labeled Organic Acid Picolinyl Esters}

To investigate the fragmentation mechanism of acyl picolinyl esters in $70 \mathrm{eV}$ EI/MS, deuterium-labeled organic acid picolinyl esters were prepared from the deuterium-labeled propionic acid, pentanoic acid, and octanoic acid $(200 \mu \mathrm{g})$ via imidazolide derivatives by the method of Balazy and Nies [13]. The picolinyl esters were reconstituted in $100 \mu \mathrm{L}$ isooctane for GC/EI/MS analysis.

\section{Transesterification}

The method of Destaillats and Angers [11] for lipids was adapted for transesterification of acylcarnitines. Briefly, a solution $(200 \mu \mathrm{L})$ containing $1 \mathrm{mg} / \mathrm{mL}$ of an acylcarnitine in methanol was evaporated to dryness under nitrogen at room-temperature. The residue was reconstituted in $1.0 \mathrm{~mL}$ anhydrous dichloromethane and reacted with a freshly prepared mixture of $100 \mu \mathrm{L}$ 1.0 $\mathrm{M}$ potassium tert-butoxide in tetrahydrofuran and $200 \mu \mathrm{L}$ 3-pyridylcarbinol. The reaction mixture was kept at $40{ }^{\circ} \mathrm{C}$ for $30 \mathrm{~min}$. After cooling to room-temperature, $2 \mathrm{~mL}$ of water and $4 \mathrm{~mL}$ of hexane were added and the solution was vortex-mixed. The organic phase was collected, passed through a disposable Pasteur pipet filled with anhydrous sodium sulfate, and the effluent was evaporated to dryness under nitrogen at room temperature. The residue was reconstituted in 100 $\mu \mathrm{L}$ isooctane for GC/EI/MS analysis.

\section{Reaction Efficiency of Transesterification Using an External Standard}

Octanoyl picolinyl ester was synthesized from octanoyl chloride. Briefly, in a capped vial, the mixture of 3-pyridylcarbinol $(6 \mathrm{mmol})$ and octanoyl chloride (5 $\mathrm{mmol}$ ) in $5 \mathrm{~mL}$ acetonitrile was stirred at room temperature for $20 \mathrm{~h}$. To the reaction solution was added $2 \mathrm{~mL}$ of $5 \%$ potassium bicarbonate and $15 \mathrm{~mL}$ hexane, followed by vortex-mixing. The hexane phase was collected, washed with $3 \times 2 \mathrm{~mL} 5 \%$ potassium bicarbonate solution and $3 \times 2 \mathrm{~mL}$ water, and dried over anhydrous sodium sulfate. The solvent was evaporated under nitrogen at room-temperature. The yield was $64 \%$ with 3.2 mmol liquid product obtained. The synthesized octanoyl picolinyl ester was more than $99 \%$ pure as determined by GC/MS, and its fragment ions were $m / z$ 92, 108, 151, 164, 178, 192, 206, 220 (molecular ion, $\mathrm{m} / \mathrm{z} 235$ ) in $70 \mathrm{eV}$ EI/MS. Undecanoyl picolinyl ester was synthesized using the same procedure as above. The product purity was also more than $99 \%$ as determined by GC/MS, and its fragment ions are $\mathrm{m} / \mathrm{z}$ 
92, 108, 151, 164, 178, 192, 206, 220, 234, 248, 262 (molecular ion, $\mathrm{m} / \mathrm{z}$ 277) in $70 \mathrm{eV} \mathrm{EI} / \mathrm{MS}$.

In five test tubes, $100 \mathrm{nmol}$ octanoylcarnitine in methanol was evaporated to dryness under nitrogen at room temperature. The transesterification was performed using the same procedure as described above, except that precisely $4.0 \mathrm{~mL}$ of the hexane-dichloromethane layer was removed for further work-up, and this fraction was accounted for in the final analysis using a calculation factor of 4/5. In another five test tubes, a calibration standard was prepared by evaporating precisely $100 \mathrm{nmol}$ octanoyl picolinyl ester in hexane to dryness under nitrogen at room temperature. Each of these 10 tubes was reconstituted using $100 \mu \mathrm{L}$ of $2.50 \times$ $10^{-4} \mathrm{M}$ undecanoyl picolinyl ester (external standard) in isooctane for GC/FID analysis. The peak area ratio of octanoyl picolinyl ester to undecanoyl picolinyl ester was calculated. The reaction efficiency was defined as the percentage of averaged peak area ratios from the octanoylcarnitine samples divided by the averaged peak area ratios from the calibration standards.

\section{Results and Discussion}

\section{Fragmentation Ion Patterns of Picolinyl Esters with Deuterium-Labeled Acyl Groups}

To investigate the fragmentation mechanism of acyl picolinyl esters, deuterium-labeled fatty acid picolinyl esters were prepared from deuterium-labeled propionic acid, pentanoic acid, and octanoic acid. The spectra of the resulting picolinyl esters are shown in Figures 1, 2, and 3.

Propionic-2,2- $\mathrm{d}_{2}$-acid picolinyl ester produces the fragment ion of $\mathrm{m} / \mathrm{z} 110$ (Figure 1a). However, propionic3,3,3- $\mathrm{d}_{3}$-acid picolinyl ester produces the fragment ion of $m / z 109$ instead of ion $m / z 110$ (Figure 1b). This is the same ion of $m / z 109$ as in the spectrum of unlabeled propionyl picolinyl ester (Figure 4a). This result is consistent with the hydrogen at $\mathrm{C} 2$ being responsible for the formation of ion $\mathrm{m} / \mathrm{z} 109$.

Fragment ion of $m / z 110$ is present in the spectrum of pentanoic-2,2- $\mathrm{d}_{2}$-acid picolinyl ester (Figure 2a), further confirming that the hydrogen at $\mathrm{C} 2$ is involved in the formation of that ion. The fragmentation of pentanoic-2,2- $\mathrm{d}_{2}$-acid picolinyl ester produces the ions of $m / z 153,165,180$, in addition to the molecular ion at $m / z 195$.

The fragmentation of the acyl group in pentanoic3,3- $\mathrm{d}_{2}$-acid picolinyl ester yields the ions of $m / z 151,166$, and 180 (Figure 2b). Pentanoic-4,4- $\mathrm{d}_{2}$-acid picolinyl ester produces the ions of 152, 164, and 180 from the cleavage of acyl group (Figure 2c). Fragment ions of $\mathrm{m} / \mathrm{z}$ 151,165 , and 178 are produced from the acyl group of pentanoic-5,5,5- $\mathrm{d}_{3}$-acid picolinyl ester (Figure $2 \mathrm{~d}$ ).

To demonstrate that the fragmentation mechanism responsible for the formation of ion $\mathrm{m} / \mathrm{z} 151$ is not primarily a McLafferty rearrangement [3], the spectrum of octanoic- $8,8,8-\mathrm{d}_{3}$ acid picolinyl ester is shown in
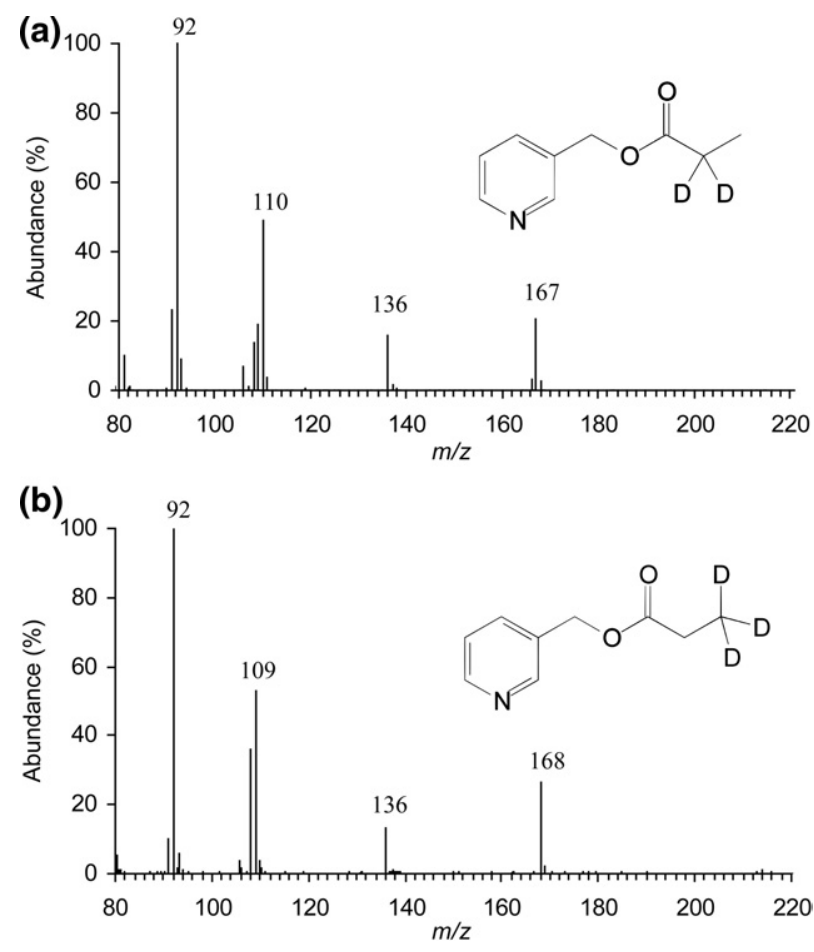

Figure 1. EI mass spectra of propionic-2,2- $\mathrm{d}_{2}$-acid picolinyl ester (a) and propionic-3,3,3- $\mathrm{d}_{3}$-acid picolinyl ester (b).

Figure 3. The fragment ions of $m / z 152$ and 193 predominate rather than the fragment ions of $\mathrm{m} / \mathrm{z} 151$ and 192, which would be expected from a McLafferty rearrangement.

\section{Mechanisms for the Formation of Fragment Ions from Acyl Picolinyl Esters}

Each acyl picolinyl ester produces fragment ions of $\mathrm{m} / \mathrm{z}$ 92 and 108. For short-chain acyl groups such as C2, C3, and C4, they also produce fragment ions of $\mathrm{m} / \mathrm{z} 109$ and 136. The fragment ions of $\mathrm{m} / \mathrm{z} 92$ and 108 are from the picolinyl moiety of picolinyl ester, and their formation mechanism has been proposed previously $[3,4,14]$.

The fragment ion of $\mathrm{m} / \mathrm{z} 93$ is present in the mass spectrum of every acyl picolinyl ester, except for the C2 acyl picolinyl ester. The signal intensity of the ion at $\mathrm{m} / \mathrm{z}$ 93 is very weak when the acyl group is three or four carbons long. However, the signal intensity of the $\mathrm{m} / \mathrm{z}$ 93 ion increases to a plateau when the major chain length of acyl group is equal to or longer than five carbons. Harvey [3] proposed that the formation of such an ion at $\mathrm{m} / \mathrm{z} 93$ resulted from the cleavage of a carbon-oxygen bond, with a hydrogen migrating from the acyl group to the nitrogen atom. Our data supports such a mechanism. The fragment ion at $\mathrm{m} / \mathrm{z} 94$ from the pentanoic acid picolinyl ester, with deuterium-labeling at different positions of the pentanoyl group, shows that the hydrogen has migrated to the nitrogen atom, and this hydrogen may come from C3, C4, or C5 (Figure 2). This is the case for short-chain acyl picolinyl esters 

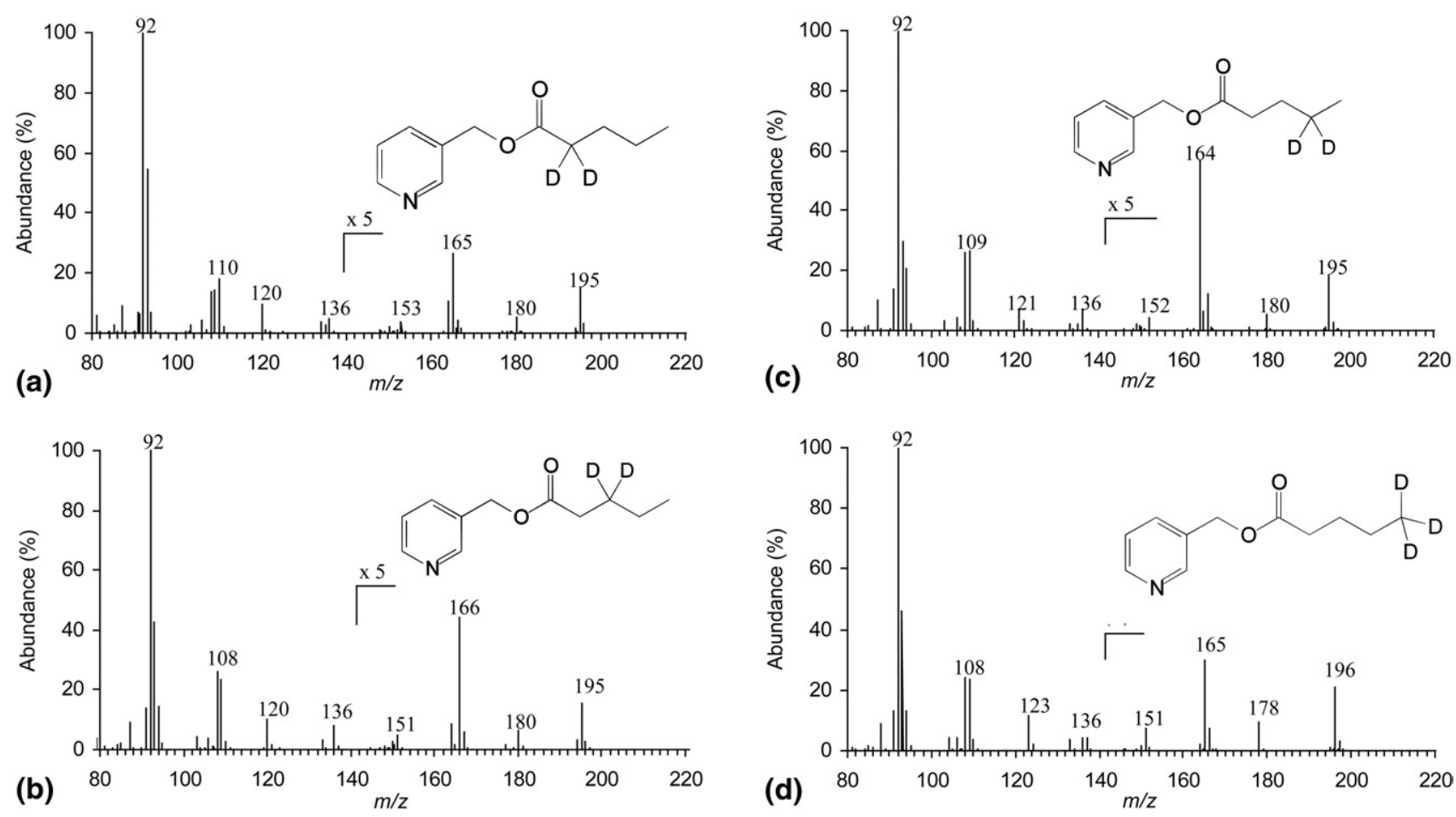

Figure 2. EI mass spectra of pentanoic-2,2- $\mathrm{d}_{2}$-acid picolinyl ester (a), pentanoic-3,3- $\mathrm{d}_{2}$-acid picolinyl ester (b), pentanoic-4,4- $\mathrm{d}_{2}$-acid picolinyl ester (c), and pentanoic-5,5,5- $\mathrm{d}_{3}$-acid picolinyl ester (s).

(short-chain means the acyl group with less than six carbons in this case). However, for medium- and longchain acyl picolinyl esters, our data indicate that the hydrogen migrating to the nitrogen atom more likely comes from the carbons beyond C4 and less likely from $\mathrm{C} 3$ and C4, and not at all from C2.

The fragment ion of $m / z 109$ is present in every acyl picolinyl ester. The signal intensity of $\mathrm{m} / \mathrm{z} 109$ ion, compared with that of $\mathrm{m} / \mathrm{z} 108$ ion, is higher in C2, C3, and C4 acyl picolinyl esters than in other acyl picolinyl esters. Thus, we propose that the formation of $\mathrm{m} / \mathrm{z} 109$ ion goes through the process depicted in Scheme 1, in which one hydrogen atom at $\mathrm{C} 2$ of the acyl group is transferred to the oxygen atom, with the two bonds breaking simultaneously. This mechanism is supported by the fragmentation patterns of propionic-2,2- $\mathrm{d}_{2}$ acid picolinyl ester and propinoic-3,3,3- $\mathrm{d}_{3}$ acid picolinyl ester (Figure 1). Propionic-2,2- $\mathrm{d}_{2}$ acid picolinyl ester produces $m / z 110$ ion in place of $m / z 109$ ion (Figure 1a), whereas propionic-3,3,3- $\mathrm{d}_{3}$ acid picolinyl ester yields $\mathrm{m} / \mathrm{z} 109$ ion (Figure $1 \mathrm{~b}$ ). The appearance of the $\mathrm{m} / \mathrm{z} 110$ ion in the spectrum of pentanoic-2,2- $\mathrm{d}_{2}$-acid picolinyl ester further supports the proposed mechanism (Figure 2a). It is very likely that the carbon-hydrogen bond at C2 must take a specific position to the carbon-oxygen during the hydrogen transfer process. The length or bulk of the acyl group may hinder the carbon-hydrogen bond moving into such specific position. This may account for the impact of acyl group length on intensity of ion at $\mathrm{m} / \mathrm{z} 109$.

The fragment ion of $m / z 151$ is present in the spectra of the acyl picolinyl esters when the backbone of the acyl group is equal to or longer than C5. Harvey [3] proposed that the $\mathrm{m} / \mathrm{z} 151$ ion was the result of a McLafferty rearrangement ( $\beta$-cleavage with concomitant specific transfer of a $\gamma$-hydrogen atom in a sixmembered transition-state in monounsaturated systems, irrespective of whether the rearrangement is based on a radical or an ionic mechanism, and irrespective of the position of the charge). However, a McLafferty rearrangement mechanism cannot explain why palmitic- $4,4-\mathrm{d}_{2}$-acid picolinyl ester also produces an ion at $\mathrm{m} / \mathrm{z} 151$ instead of at $\mathrm{m} / \mathrm{z} 152[4,5]$. Based on the observation that the deuterium at $\mathrm{C} 4$ of pentanoic-4,4$\mathrm{d}_{2}$-acid picolinyl ester migrates to the nitrogen atom $(\mathrm{m} / \mathrm{z} 94$ ion is found), we propose the structure of $\mathrm{m} / \mathrm{z}$

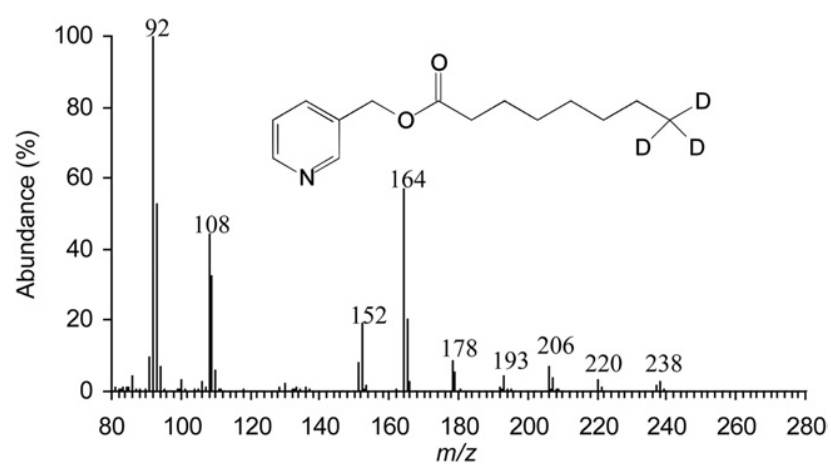

Figure 3. EI mass spectrum of octanoic-8,8,8-d3-acid picolinyl ester. 

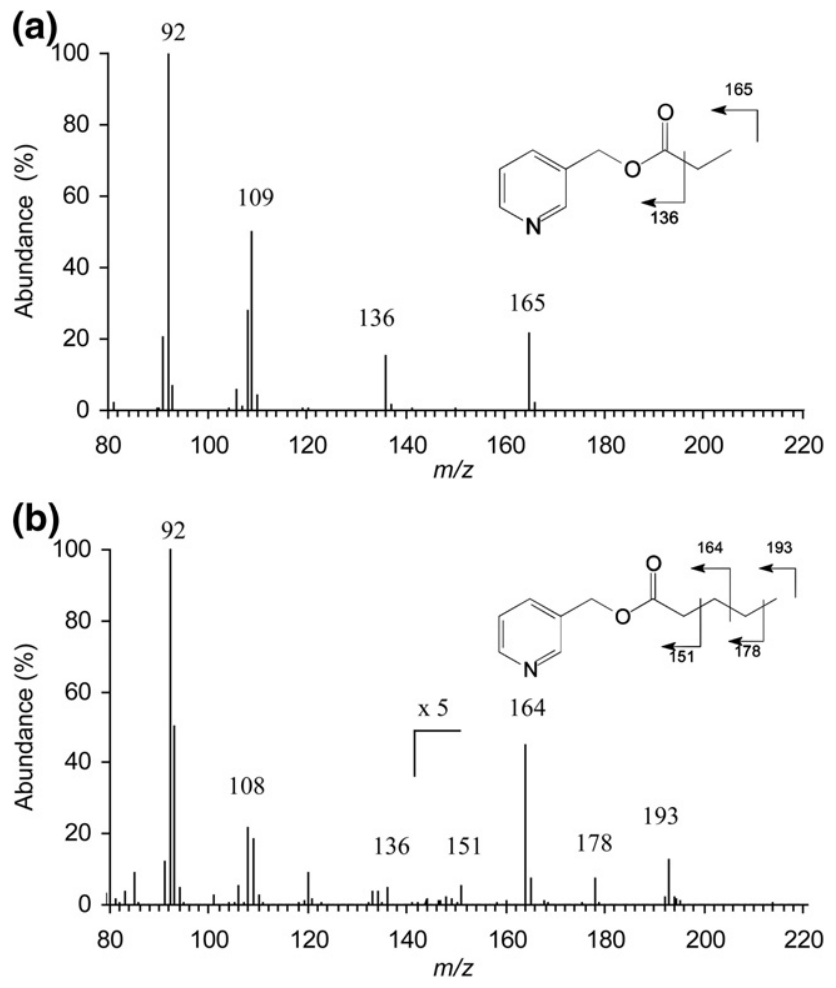

Figure 4. EI mass spectra of propionyl (a) and pentanoyl (b) picolinyl esters derived from acylcarnitines following their transesterification.

151 ion shown in Scheme 2a. This structure is very stable because the radical site at $\mathrm{C} 2$ is conjugated with the double-bond of an ester. A similar distonic radical cation, in which a radical charge is stabilized through conjugation with a double-bond, has been reported [15]. The low signal intensity of $\mathrm{m} / \mathrm{z} 151$ ion in pentanoic acid picolinyl ester is possibly due to the difficulty in forming an intermediate with a radical site at C4. For medium- and long-chain acyl groups, the formation of the intermediate with a radical site at $\mathrm{C} 4$ would be the product resulting from the exchange of the hydrogen at $\mathrm{C} 4$ with the radical site at $\mathrm{C} 7$ or $\mathrm{C} 8$ through a five- or six-membered transition-state. There are two possible dissociation pathways for the intermediate with the radical site at $\mathrm{C} 4$, resulting in two different products: (1) production of the ion at $\mathrm{m} / \mathrm{z} 151$ (Scheme 2a); (2) production of the ion at $m / z 192$ by the same pathway as the formation of $\mathrm{m} / \mathrm{z} 164$ and 178 ion (Scheme $\mathbf{2 b}$ and 2c). The fragment ion of $\mathrm{m} / \mathrm{z} 151$ is more stable than the fragment ion of $\mathrm{m} / \mathrm{z} 192$ because of its conjugated structure. This mechanism is further supported by the fragment ions of octanoic-8,8,8- $\mathrm{d}_{3}$-acid picolinyl ester (Figure 3), in which fragment ions of m/z 152 and 193 are observed. This mechanism also can explain the fragment ion at $m / z 151$ from palmitic-4,4- $\mathrm{d}_{2}$-acid picolinyl ester $[4,5]$ and the fragment ions at $m / z 151,152$, or 153 from deuterium-labeled pentanoic acid picolinyl esters (Figure 2) (those fragment ions at $\mathrm{m} / \mathrm{z} 151,152$ or 153 from deuterium-labeled pentanoic acid picolinyl esters are interpretable by a McLafferty rearrangement mechanism). Moreover, this mechanism suggests the reason for the relatively weak intensity of ion $m / z 192$, and the very abundant intensity of ion $\mathrm{m} / \mathrm{z} 151$ in medium-chain and long-chain acyl picolinyl esters.

Based on the observation that the fragment ions of $\mathrm{m} / \mathrm{z} 164$ and 178 begin to appear only when the acyl group length is $\mathrm{C} 5$ or longer, the formation of these ions from pentanoic picolinyl ester is consistent with the proposed processes in Scheme $\mathbf{2 b}$ and $\mathbf{2 c}$. This mechanism is supported by the fragment ion patterns of deuterium-labeled pentanoic acid picolinyl ester (Figure 2). The mechanism involves charge-site formation on the nitrogen atom, rather than on the oxygen atom. A hydrogen atom at $\mathrm{C} 3$ or $\mathrm{C} 4$ or $\mathrm{C} 5$ would then transfer to the nitrogen atom, with the positive charge localized to the nitrogen and a radical electron on the methylene carbons $\mathrm{C} 3$ or $\mathrm{C} 4$ or $\mathrm{C} 5$. The radical site at $\mathrm{C} 5$ is then transferred to $\mathrm{C} 2$ through hydrogen exchange via a five-membered transition-state. The $\beta$-cleavage, happening at the position adjacent to the radical position, produces the ions of $m / z 164$ and 178. Compared with the ion of $\mathrm{m} / \mathrm{z} 178$, the ion of $\mathrm{m} / \mathrm{z} 164$ is more stable due to the conjugated double bonds, suggesting why the signal for $\mathrm{m} / \mathrm{z} 164$ is more intense. This formation mechanism of the ion at $\mathrm{m} / \mathrm{z} 164$ is not in agreement with that proposed by Harvey [3], because our data show that the hydrogen at $\mathrm{C} 2$ does not directly migrate to the nitrogen atom. The structure herein proposed for the ion at $m / z 164$ is similar to that proposed by Christie [4], except that the hydrogen migrates to the nitrogen and not to the oxygen atom. This is supported by the $\mathrm{m} / \mathrm{z} 94$ ion observed in the fragment ions of deuteriumlabeled pentanoic acid picolinyl esters (Figure 2). For acyl groups longer than $\mathrm{C} 5$, our data suggest that a radical site at C2, C3, or C4 is formed more likely by hydrogen exchange with a radical site on the methylene carbons C6, C7, or C8 through a five- or six-membered transition-state, because the hydrogen at $\mathrm{C} 3$ or $\mathrm{C} 4$ appears to be less likely to migrate to the nitrogen atom directly, suggested by low peak intensities of $\mathrm{m} / \mathrm{z} 93$ ion signal in propionyl (C3) and butyryl (C4) picolinyl

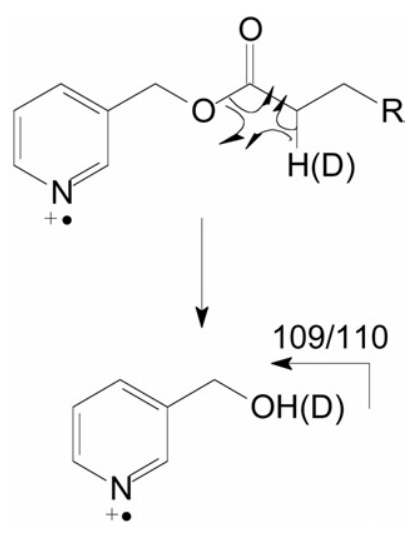

Scheme 1. Proposed mechanism for the formation of fragment ion at $m / z 109$. 


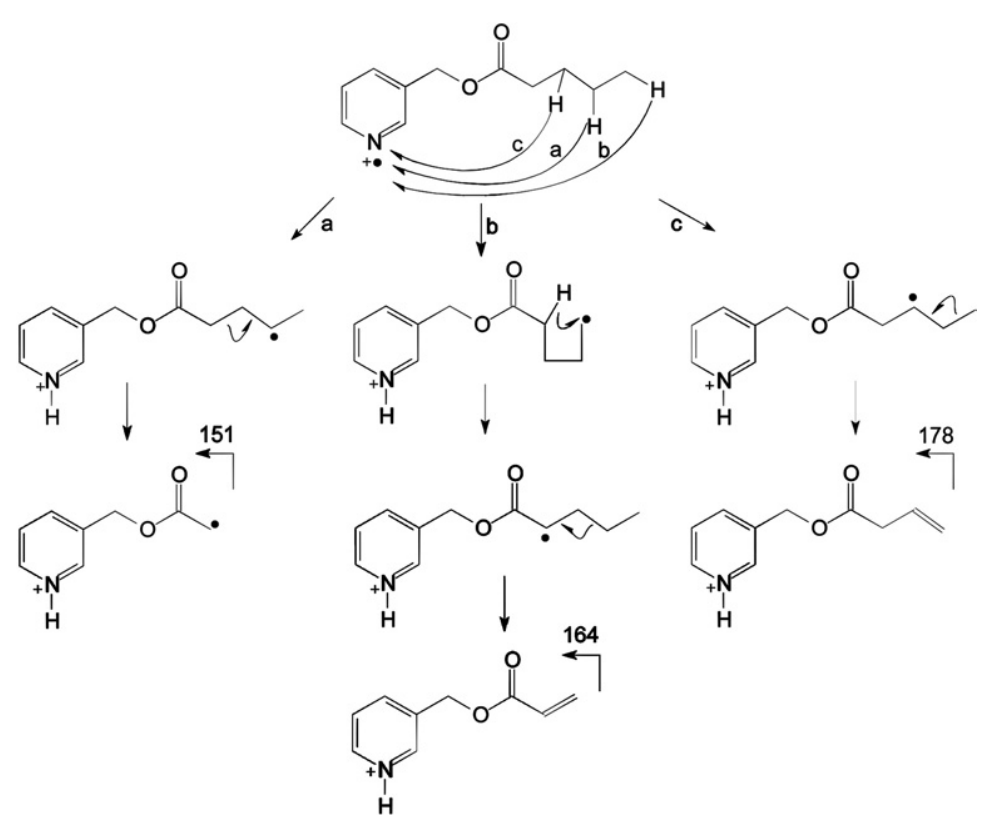

Scheme 2. Proposed mechanism for the formation of fragment ions at $m / z 151,164$, and 178 from pentanoic acid picolinyl ester.

esters, and no $\mathrm{m} / \mathrm{z} 93$ in acetyl (C2) picolinyl ester. Moreover, the aforementioned formation mechanism indicating a radical site at $\mathrm{C} 2, \mathrm{C} 3$, or C4 for acyl groups longer than $\mathrm{C} 5$ is further supported by the fragment ion pattern of octanoic-8,8,8- $\mathrm{d}_{3}$-acid picolinyl ester (Figure 3), in which ions at $m / z 152,165$ and 179 are observed.

\section{Transesterification of Acylcarnitines}

Acyl picolinyl esters have been applied to characterize the acyl group structures of fatty acids, lipids, and phospholipids from cell membranes [16]. Most of these fatty acids belong to the class of long-chain acyl groups, such as saturated or unsaturated $\mathrm{C} 16$ and C18 fatty acids. The acyl picolinyl esters usually produce a series of fragment ions from the sequential cleavage of the acyl group, and the fragment ion pattern has been successfully employed to characterize the acyl group's chemical structure. Accordingly, the positions of double bonds and branched methyl groups within the acyl group can be determined with EI/MS. However, the synthesis of acyl picolinyl esters usually starts from fatty acids. For lipids or phospholipids, there are two steps to arrive at the corresponding picolinyl ester, the first being hydrolysis to form its fatty acid. Libert et al. [8-10] used the above method to characterize the acyl group structures of some acylcarnitines in normal human urine.

Transesterification [11], reacting lipid directly with 3-pyridylcarbinol under mild conditions, has been shown to be a successful method for synthesis of the corresponding picolinyl ester in one step. In the present study, the transesterification method was applied to the synthesis of acyl picolinyl esters from the corresponding acylcarnitines. In the transesterification reaction to form acyl picolinyl esters, the acyl group from the acylcarnitine is transferred to 3-pyridylcarbinol by the action of potassium tert-butoxide (Scheme 3). Acylcarnitines, with acyl group lengths ranging from short- to long-chain, were transesterified to produce their corresponding acyl picolinyl esters. These esters were then separated from each other by capillary GC. All acylcarnitines examined in this study successfully formed their corresponding acyl picolinyl esters. Each acyl picolinyl ester yielded a unique EI/MS spectrum as a function of the acyl group. Most of the chemical structures of the acyl groups from these acylcarnitines can be deduced from their fragmentation ion patterns under $70 \mathrm{eV} \mathrm{EI/MS}$ conditions.

To investigate the reaction efficiency of the transesterification, octanoylcarnitine (C8) was selected as the exemplar. To perform this study, octanoyl (C8) picolinyl ester and undecanoyl (C11) picolinyl ester were synthesized to serve as comparison standards. The transesterification reaction efficiency of octanoylcarnitine (C8) was determined to be $82 \pm 5 \%(n=5)$. In another experiment, with variable reaction times (data not shown), the transesterification efficiency reached a plateau after $5 \mathrm{~min}$ and

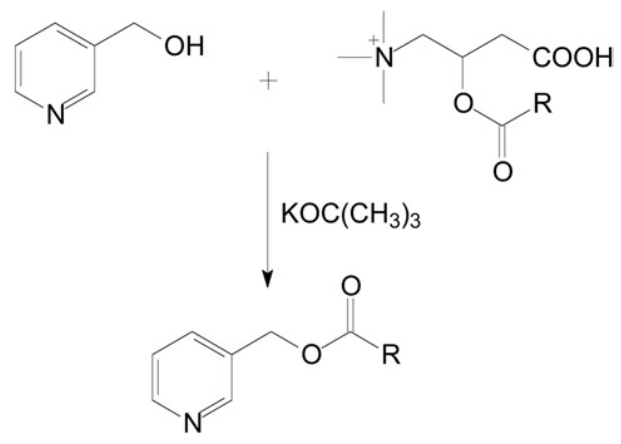

Scheme 3. Transesterification reaction of acylcarnitines. 
remained constant through $55 \mathrm{~min}$. Consequently, a reaction time of $30 \mathrm{~min}$ was used for the transesterification of all acylcarnitines in the present experiments.

\section{Fragmentation Patterns of Picolinyl Esters Derived from Transesterified Acylcarnitines with Short-Chain Acyl Groups}

The fragment ion patterns of short-chain acyl picolinyl esters derived from transesterified acylcarnitines are illustrated by the EI/MS spectra of propionyl and pentanoyl picolinyl esters (Figure 4). For propionyl picolinyl ester, the EI/MS spectrum displays the molecular ion of $m / z 165$ and fragment ions of $m / z 92,108,109$, and 136. The latter peaks $(\mathrm{m} / \mathrm{z} 92,108$, and 109) are all characteristic of the picolinyl moiety. The $\mathrm{m} / \mathrm{z} 136$ ion is produced from the scission of the carbon-carbon bond adjacent to the carbonyl group (Figure 4a). The signal intensity of the $\mathrm{m} / \mathrm{z} 136$ ion is remarkable only in the spectra of short-chain acyl picolinyl esters. Both acetyl and butyryl picolinyl esters, similar to propionyl picolinyl ester, show their molecular ion peaks and the peak of $m / z 136$ ion (data not shown). The pentanoyl picolinyl ester produces not only fragment ions of $m / z 92$ and 108 from the picolinyl moiety, but also fragment ions of $\mathrm{m} / \mathrm{z}$ 151, 164, 178, and 193 from the sequential cleavage of the acyl group (Figure $4 b$ ). From the fragment ions in the mass spectrum, apparently the methylene groups from the pentanoyl moiety are cleaved sequentially. We have found that the acyl group of picolinyl esters show this pattern of fragment ions when there are at least five carbons in the backbone of the acyl moiety.

\section{Fragmentation Patterns of Picolinyl Esters Derived from Transesterified Acylcarnitines with Medium- Chain Acyl Groups}

The fragmentation of the medium-chain acyl picolinyl esters derived from acylcarnitines is illustrated by the EI/MS spectra of nonanoyl and 4-methyl-octanoyl picolinyl esters (Figure 5). The two compounds are constitutional isomers with the same molecular mass $(\mathrm{m} / \mathrm{z}$ 249). 4-Methyl-octanoyl picolinyl ester elutes from the GC capillary column earlier than nonanoyl picolinyl ester (for all acyl picolinyl esters observed, the isomers with branched-chains had shorter retention times than their straight-chain isomers). As expected, nonanoyl picolinyl ester produces fragment ions of $m / z$ 151, 164, $178,192,206,220,234$, and 249 from the sequential cleavage of the straight-chain acyl moiety (Figure 5a). 4-Methyl-octanoyl picolinyl ester produces the same fragment ions ( $m / z$ 151, 164, 192, 206, 220, 234, and 249, Figure $5 b$ ) as the nonanoyl picolinyl ester, but the fragment ion peak intensities are different from those of nonanoyl picolinyl ester. Both the relatively less intense signal of $\mathrm{m} / \mathrm{z} 178$ and a gap of 28 between ions of $\mathrm{m} / \mathrm{z} 164$ and 192 (Figure 5b) are seen as evidence that there is a branched methyl group at C4 of the acyl group.
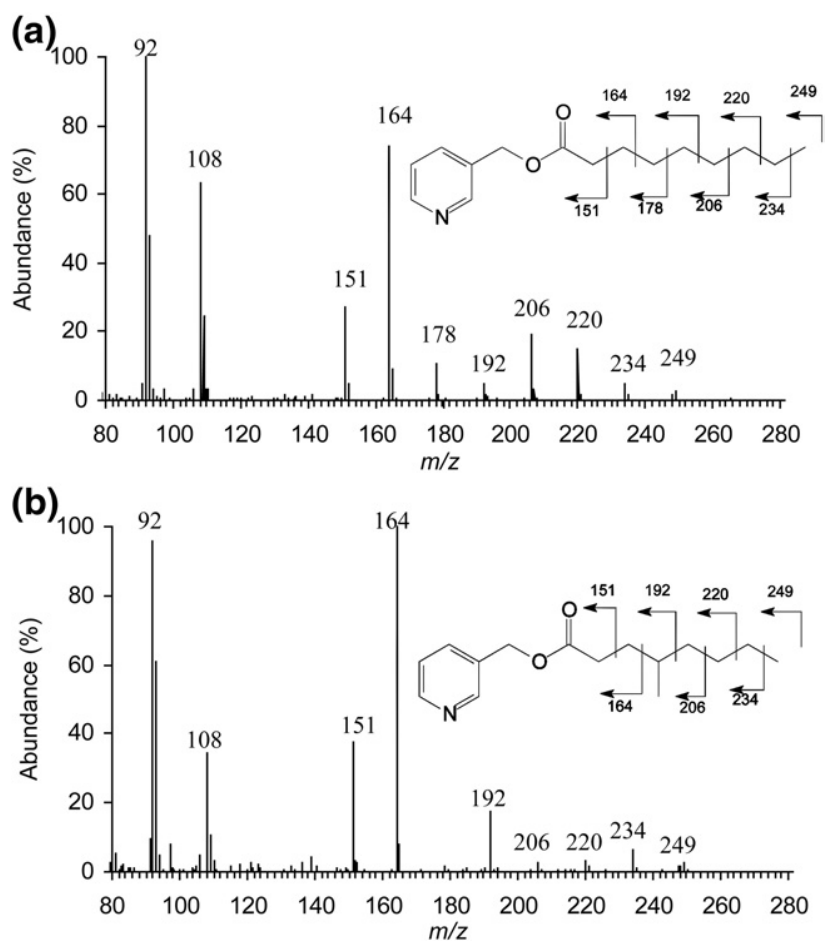

Figure 5. EI mass spectra of nonanoyl (a) and 4-methyl-octanoyl (b) picolinyl esters derived from acylcarnitines following their transesterification.

For medium-chain acyl picolinyl esters, the fragmentation pattern of the methyl-branched acyl groups is reduced in intensity (Figure 5) when compared with that of straight-chain acyl picolinyl esters. The methylbranched acyl groups may hinder the process of hydrogen transfer from the acyl group to the nitrogen atom. In the spectrum of 4-methyl-octanoyl picolinyl ester, the enhanced abundance of $\mathrm{m} / \mathrm{z} 192$ presumably reflects the stability of that ion because there is a methyl group at the end of the double-bond.

\section{Fragmentation Patterns of Picolinyl Esters Derived from Transesterified Acylcarnitines with Long- Chain Acyl Groups}

For long-chain acylcarnitines, the fragmentation patterns of their picolinyl esters are illustrated by the EI/MS spectra of palmitoyl and 2-methyl-hexadecanoyl picolinyl esters (Figure 6). As anticipated, the sequential cleavage of the acyl moiety of palmitoyl picolinyl ester produces the fragment ions of $\mathrm{m} / \mathrm{z} 151,164,178,192$, 206, 220, 234, 248, 262, 276, 290, 304, 318, 332, and 347 (Figure 6a), all of which differ by $14 \mathrm{u}$ for all of the ions from $[\mathrm{M}-15]^{+}$ion to $\mathrm{m} / \mathrm{z} 164$ ion. Based on the signal intensities and $\mathrm{m} / \mathrm{z}$ values of the above fragment ions, it is reasonable to conclude that the acyl group has a straight chain structure. For the 2-methyl-hexadecanoyl picolinyl ester (Figure 6b), its acyl moiety cleavage produces two abundant peaks of $\mathrm{m} / \mathrm{z} 165$ and 178 instead of $m / z 151$ and 164, due to the methyl group at 

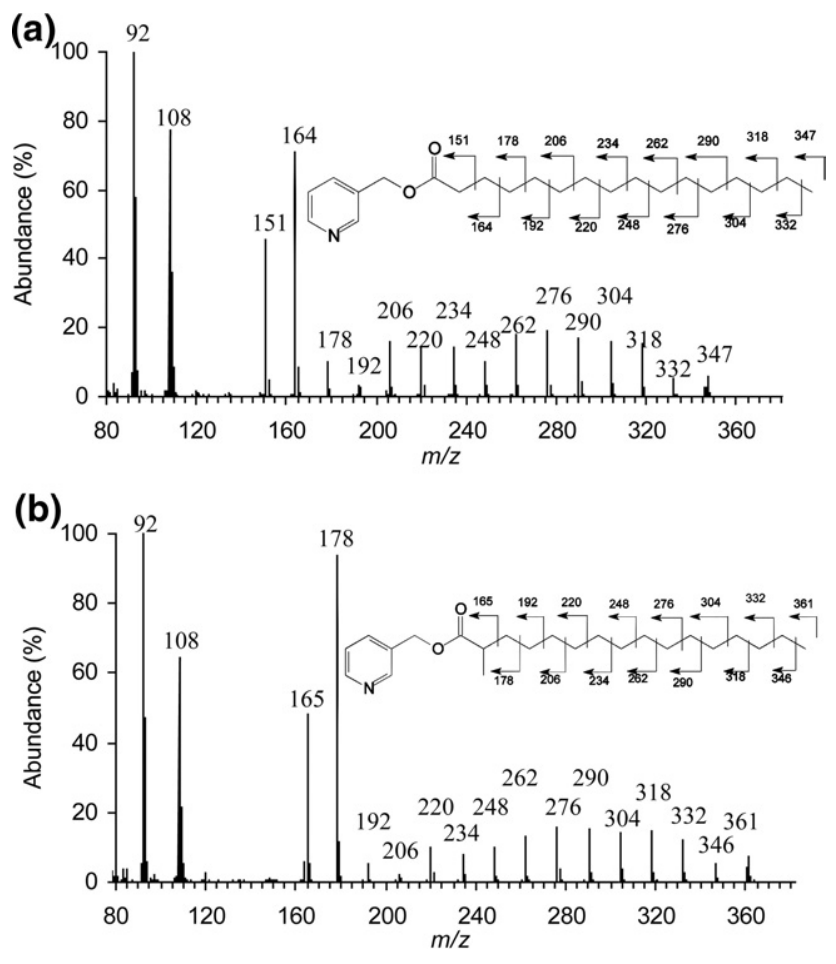

Figure 6. EI mass spectra of palmitoyl (a) and 2-methyl-hexadecanoyl (b) picolinyl esters derived from acylcarnitines following their transesterification.

C2 of the acyl moiety. The other fragment ions are similar to those of palmitoyl picolinyl ester because they emanate from the sequential cleavage of the straight chain part of the acyl group. More abundant peaks of $\mathrm{m} / \mathrm{z} 165$ and 178 were observed with other 2-methyl acyl picolinyl esters, such as 2-methyl octanoyl picolinyl ester [8]. Thus, the peaks of $m / z 165$ and 178 can be used to identify a methyl group at C2 of an acyl group of an acyl picolinyl ester. The successful formation of 2-methyl-hexandecanoyl picolinyl ester in this transesterification method shows that the 2-methyl group on the acyl part of acylcarnitines does not interfere with the transesterification reaction of an acylcarnitine with 3-pyridylcarbinol.

\section{Fragmentation Patterns of Picolinyl Esters Derived from Transesterified Acylcarnitines with Unsaturated Acyl Groups}

For unsaturated acylcarnitines, the fragmentation patterns of their acyl picolinyl esters are illustrated by the EI/MS spectra of trans-2-dodecenoyl and cis-4-octenoyl picolinyl esters (Figure 7). The trans-2-dodecenoyl picolinyl ester yields fragment ions of $m / z 151,164,177$, 190, 204, 218, 232, 246, 260, 274, and 289 from the sequential cleavage of the acyl group (Figure 7a). The signal intensities of $\mathrm{m} / \mathrm{z} 177$ and 190 are much greater than are those of other peaks. This phenomenon was also observed in other trans-2-acyl picolinyl esters, including trans-2-octenoyl, trans-2-decenoyl, trans-2- tetradecenoyl, and trans-2-hexadecenoyl picolinyl esters (data not shown). Therefore, the presence of $m / z 177$ and 199, as well as their relative intensities, can be used to identify a double-bond between $\mathrm{C} 2$ and $\mathrm{C} 3$ in the acyl groups of acylcarnitines. Cis-4-octenoyl picolinyl ester produces the fragment ions of $m / z 151,164,190,204$, 218 , and 233 from the sequential cleavage of its acyl group (Figure $7 \mathrm{~b}$ ). The difference of $26 \mathrm{u}$ between $\mathrm{m} / \mathrm{z}$ 164 and 190 is consistent with a double-bond between C4 and C5 in the octenoyl group. Trans-4-octenoyl picolinyl ester also yields the fragment ions of $\mathrm{m} / \mathrm{z} 151$, 164, 190, 204, 218, and 233 (data not shown), and their abundances are identical to those of cis-4-octenoyl picolinyl ester. Therefore, this method can determine the positions of branched methyl groups and double bonds, but not the cis- and trans-configurations of a doublebond in the acyl group.

For unsaturated acyl picolinyl esters, the fragmentation patterns of trans-2 acyl picolinyl esters (Figure 7a) contain a unique peak of $\mathrm{m} / \mathrm{z} 177$. The same $\mathrm{m} / \mathrm{z} 177$ ion has been reported in the fragment ion pattern of cis-2tetradecenoic acid picolinyl ester [17]. We propose that the structure of $\mathrm{m} / \mathrm{z} 177$ ion is analogous to that of $\mathrm{m} / \mathrm{z}$ 151 ion (as seen in Scheme 2a), with the radical electron at C4 stabilized through conjugation with the two conjugated double bonds. This may account for the enhanced abundance of that peak. The enhanced peak signal at $m / z 190$ also may be due to the stability of three conjugated double bonds. We observe that the mediumchain unsaturated acyl picolinyl esters show reduced
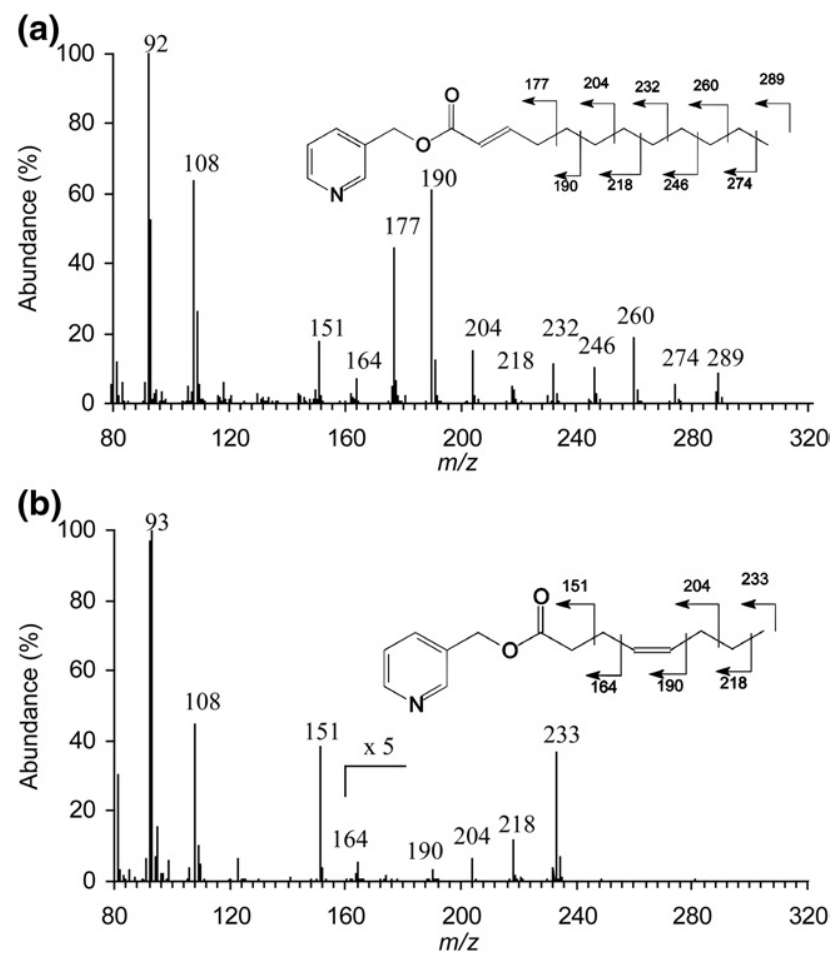

Figure 7. EI mass spectra of trans-2-dodecenoyl (a) and cis-4octenoyl (b) picolinyl esters derived from acylcarnitines following their transesterification. 
peak intensities of fragment ions from the acyl group (Figure $7 \mathrm{~b}$ ). No peak is found between $\mathrm{m} / \mathrm{z} 164$ and 190 in cis-4-octenoyl picolinyl ester, a circumstance that is different from the fragmentation patterns of the longchain unsaturated acyl picolinyl esters [2]. However, a space of $26 \mathrm{u}$ between $\mathrm{m} / \mathrm{z} 164$ and 190 is consistent with a double-bond at C4 in the acyl group.

\section{Conclusions}

We have proposed mechanisms for the formation of fragment ions of acyl picolinyl esters based on studies using deuterium-labeled acyl groups and short-, medium-, and long-chain acyl groups. We have demonstrated that the picolinyl ester derivatives of acylcarnitines can be efficiently prepared in one step by transesterification under mild conditions. We have shown that the chemical structures of the acyl groups of acylcarnitines, including the positions of double-bond and branched methyl groups, can be determined from the fragment ions obtained under $70 \mathrm{eV} \mathrm{EI} / \mathrm{MS}$ condition.

\section{Acknowledgments}

This work is supported in part by the NIH Road Map grant R33DK270291, NIH grant P01AG15885, and the Department of Veterans Affairs Medical Research Service. The authors thank Steve Ingalls for reviewing the mass spectrometry data and Bernard Tandler, Ph.D., for editorial help.

\section{References}

1. Harvey,D. J. Picolinyl Esters for the Structural Determination of Fatty Acids by GC/MS. Mol. Biotechnol. 1998, 10(3), 251-260.
2. Dobson, G.; Christie, W. W. Structural Analysis of Fatty Acids by Mass Spectrometry of Picolinyl Esters and Dimethyloxazoline Derivatives. Trends Anal. Chem. 1996, 15, 130-136.

3. Harvey, D. J. Picolinyl Ester as Derivatives for the Structural Determination of Long Chain Branched and Unsaturated Fatty Acids. Biomed. Mass Spectrom. 1982, 9, 33-38.

4. Hamilton, J. T. G.; Christie W. W. Mechanism for Ion Formation During the Electron Impact-Mass Spectrometry of Picolinyl Ester and 4,4Dimethyloxazoline Derivatives of Fatty Acid. Chem. Phys. Lipids 2000, 105, 93-104.

5. http://www.lipidlibrary.co.uk/ms/arch_pic/pi_sat/Pi0033.gif

6. Lowes, S.; Rose, M. E. Simple and Unambiguous Method for Identifying Urinary Acylcarnitines Using Gas Chromatography-Mass Spectrometry. Analyst 1990, 115, 511-516.

7. Lowes, S.; Rose, M. E.; Mills, G. A.; Pollitt, R. J. Identification of Urinary Acylcarnitines Using Gas Chromatography-Mass Spectrometry: Preliminary Clinical Applications. J. Chromatogr. 1992, 577(2), 205-214.

8. Libert, R.; Van Hoof, F.; Thillaye, M.; Vincent, M. F.; Nassogne, M. C.; Stroobant, V.; Hoffmann, E. D.; Schanck, A. Identification of New Medium-Chain Acylcarnitines Present in Normal Human Urine. Anal. Biochem. 1997, 251, 196-205.

9. Libert, R.; Van Hoof, F.; Thillaye, M.; Vincent, M. F.; Nassogne, M. C.; Stroobant, V.; de Hoffmann, E.; Schanck, A. Identification of New Medium-Chain Acylcarnitines Present in Urine of a Patient with MediumChain Acyl-CoA Dehydrogenase Deficiency. J. Inherit. Metab. Dis. 1999, 22(1), 9-18.

10. Libert, R.; Van Hoof, F.; Thillaye, M.; Vincent, M. F.; Nassogne, M. C.; de Hoffmann, E.; Schanck, A. Identification of Undescribed Medium-Chain Acylcarnitines Present in Urine of Patients with Propionic and Methylmalonic Acidemias. Clin. Chim. Acta. 2000, 295(1-2), 87-96.

11. Destaillats, F.; Angers, P. One-Step Methodology for the Synthesis of FA Picolinyl Esters from Intact Lipids. J. Am. Oil Chem. Soc. 2002, 79, 253-256.

12. Ziegler, H. J.; Bruckner, P.; Binon, F. O-Acylation of dl-Carnitine Chloride. J. Org. Chem. 1967, 32(12), 3989-3991.

13. Balazy, M.; Nies, A. S. Characterization of Epoxides of Polyunsaturated Fatty Acids by Mass Spectrometry via 3-Pyridinylmethyl Esters. Biomed. Environ. Mass Spectrom. 1989, 18(5), 328-36.

14. Harvey, D. J. Picolinyl Derivatives for the Characterization of Cyclopropane Fatty Acids by Mass Spectrometry. Biomed. Mass Spectrom. 1984, 11, 187-192.

15. Hiserodt R. D.; Pope B. M.; Cossette M.; Dewis M. L. Proposed Mechanisms for the Fragmentation of Doubly Allylic Alkenamides (Tingle Compounds) by Low Energy Collisional Activation in a Triple Quadrupole Mass Spectrometer. J. Am. Soc. Mass Spectrom. 2004, 15(10), 1462-1470.

16. Wing, D. R.; Harvey, D. J.; La Droitte, P.; Robinson, K.; Belcher, S. Examination of the Esterified Fatty Acids from Mouse Erythrocyte and Synaptosomal Membrane Phospholipids and Their Distribution Between the Various Phospholipid Types. J. Chromatogr. 1986, 368, 103-111.

17. Kurkiewicz, S.; Dzierzewicz, Z.; Wilczok, T.; Dworzanski, J. P. GC/MS Determination of Fatty Acid Picolinyl Esters by Direct Curie-Point Pyrolysis of Whole Bacterial Cells. J. Am. Soc. Mass Spectrom. 2003, 14, 58-62. 